\title{
Perceived Health Risks of Exposure to Indoor Air Pollution from Cooking Fuels in Sindh, Pakistan
}

\author{
Sohail Ahmed Rajper ${ }^{1}$, Adnan Nazir ${ }^{2}$, Sana Ullah ${ }^{1 * *}$, Zhongqiu Li ${ }^{1 *}$ \\ ${ }^{1}$ School of Life Sciences, Nanjing University, Nanjing, Jiangsu, P.R. China \\ ${ }^{2}$ Department of Agricultural Economics, Sindh Agriculture University, Tando Jam, Pakistan
}

Received: 21 May 2019

Accepted: 1 September 2019

\begin{abstract}
Use of solid fuel for cooking is one of the major sources of household indoor air pollution, especially in developing countries. Around 3 billion people still cook and heat their homes using open fires and leaky stoves, burning biomass fuel, i.e., wood, crop residues, animal dung, wood charcoal and coal as their primary source of domestic energy. The present study was conducted to assess the perceived level of health risks in rural households associated with indoor air pollution caused by cooking (cleaner and solid) fuels. For this purpose, the data was collected from 700 rural households from 5 districts of Sindh Province, Pakistan. Using the Probit regression model, factors such as household attributes, cooking activities, behavioral health effects, physical health effects, and regional factors were controlled. The results indicate that solid fuels revealed a positive effect on the factors used in the models. In addition, the pseudo- $R^{2}$ values indicate the goodness of fit of the models, which varied from 0.16 to 0.38 . Which depicts a good fit of our models in explaining the perceived risk of physical and behavioral effects by polluting indoor air through solid fuels used by households. Furthermore, there is a need for strong policy-making and public awareness programs to mitigate the exposure and associated health risks of indoor air pollution. Therefore, public and private organizations and academia working on environmental health should develop an action plan for reducing indoor air pollution for the future.
\end{abstract}

Keywords: solid fuels, cleaner fuels, indoor air pollution, probit regression, Pakistan

\section{Introduction}

About $50 \%$ of the world's low-income population uses biomass fuel as their primary source for cooking [1], while the usage is even higher in developing countries. The burning of biomass fuel emits pollutants into the air, which are detrimental to human health. Some of those pollutants are suspended particulate

*e-mail: lizq@nju.edu.cn,

**e-mail: sunyuop@gmail.com matter, carbon monoxide, polyaromatic hydrocarbons, formaldehyde, and volatile organic compounds [2]. These pollutants can cause many health diseases in humans, specifically respiratory problems such as chronic bronchitis [3] and bronchial asthma, and can cause tuberculosis and abnormalities in newborns [4, 5]. The use of solid fuels for cooking in rural areas of developing countries cannot be denied because it is used in many forms such as coal, charcoal, wood, straw, dung cakes, and agricultural crop residues [3]. Furthermore, due to limited alternative sources of fuel in rural areas of Pakistan people are heavily relying on solid fuel for cooking. Mostly women and children 
are at higher risk because they spend more time in the kitchen in rural areas, as women are mainly involved in cooking. It has been observed that indoor pollution is the eighth most important risk factor responsible for the global burden of disease [6].

Pakistan is currently the fifth most populous country in the world, located in northwest south Asia [7], with an average household size of 6.45 persons. Pakistan is an agricultural country, and nearly $62.5 \%$ of the population live in rural areas [8], where wood, straw/ shrubs/grass, animal dung, charcoal, and coal are the only available options for domestic cooking fuel $[9,10]$. It is estimated that $74.4 \%$ of households in rural and $11.9 \%$ of urban households in Pakistan use solid fuels for cooking [11], and biomass fuel is burnt in open fires, producing health-damaging pollutants and chemicals associated with chronic bronchitis $[6,12]$.

A study carried out in Pakistan depicted that the use of solid fuel cooking in the kitchen may increase particulate matter $\left(\mathrm{PM}_{10}\right)$ concentrations up to $8,555 \mu \mathrm{g} / \mathrm{m}^{3}$ [13]. Estimates indicate that IAP causes over 2.854 million deaths globally [14]. In Pakistan, biomass fuel is used for about $67 \%$ of the cooking throughout the country [15]. In rural areas of Sindh Province of Pakistan, kitchens are not properly ventilated and therefore the effects of the indoor air pollutants of solid fuels may be even worse. Many studies regarding the effect of indoor air pollution have been conducted in the past in developing as well as developed countries [16, 17], and detrimental effects of solid fuels have been observed as well $[3,5]$. However, most of these studies were either conducted in one of a few locations or were based on the effect shown on a limited number of individuals within a region.

The present study is based on indirect exposure assessment, which reduces the strength of causality. Using cooking fuel as a proxy for pollution exposure ignores the causal linkage between smoke inhalation and its impact on respiratory health. Especially in rural areas, numerous households do not consume a single type of cooking fuel; rather they use a mixture of fuels for their cooking requirements. The objective of this paper is, therefore, to perceive the exposure to indoor air pollution by determining the proximate determinants of fuel choice in the Sindh Province of Pakistan. No investigation regarding fuel choice and perceived health effects has been undertaken so far, and very few works on indoor pollution and fuel usage have been done in Pakistan.

\section{Materials and Methods}

\section{Study Area}

In the present study, for investigating perceived household health risks due to indoor air pollution while using cooking fuels in Pakistan, Sindh Province was selected because $51.25 \%$ of households live in rural areas [8]. Sindh is one of the five provinces of Pakistan, in the southeastern part of Pakistan, and is the $2^{\text {nd }}$ largest province by a population at 47.89 million and the $3^{\text {rd }}$ largest by area at $140,914 \mathrm{~km}^{2}$. Sindh ranks second among Pakistani provinces in its GDP of 83.71 million

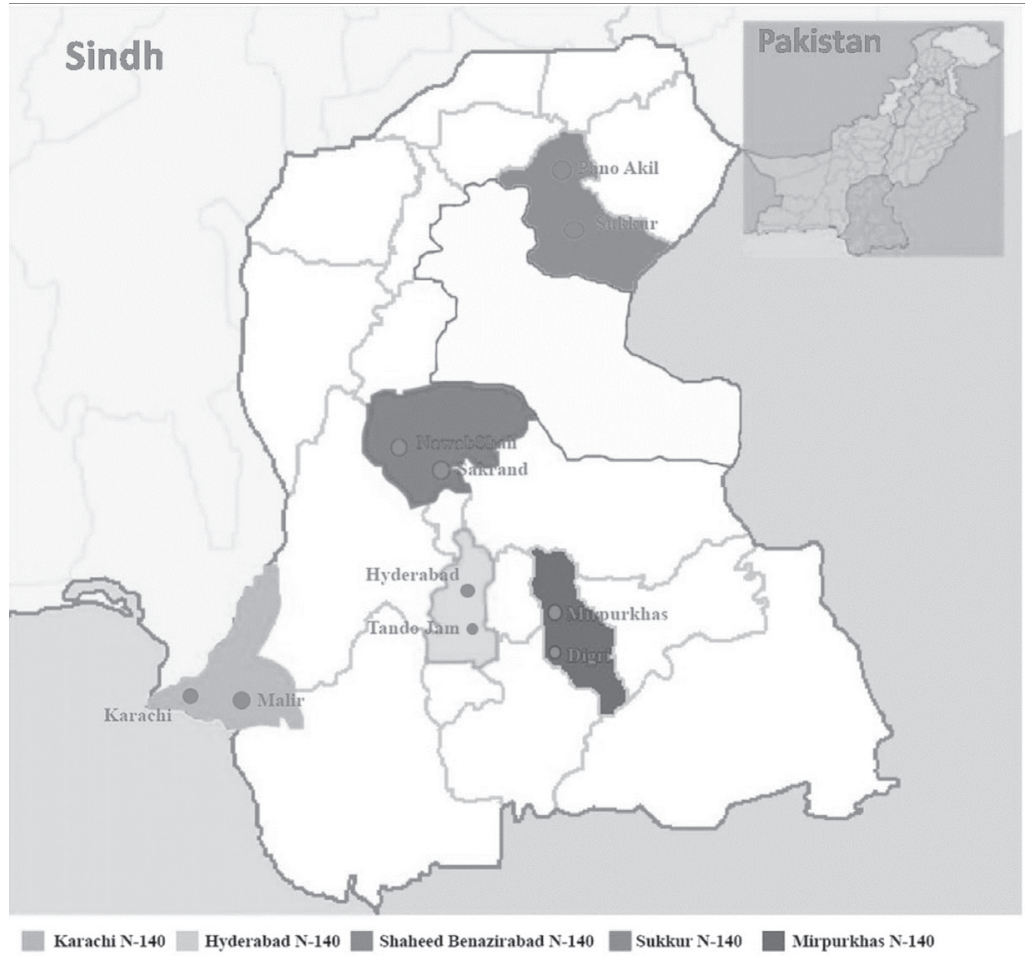

Fig. 1. Geographical locations of the five districts covered in the intervention. 
USD [18]. More than half of the population are rural dwellers in the suburbia settings of Karachi, Hyderabad, Mirpur Khas, Shaheed Benazirabad, and Sukkur.

For this study, Sindh was selected as its climatic conditions are hot and dry, due to which it is positioned in the subtropical arid region. Because of its sedimentary plain it is categorized into 3 regions, namely upper, central and lower. In the upper region, the maximum temperature can hit more than $50^{\circ} \mathrm{C}$. Similarly, in the winters the temperature commonly drops to $6^{\circ} \mathrm{C}$. From Hyderabad to Sukkur districts are known as the central region, where the temperature is more than the lower region and less than the upper region. The lower region is highly humid and stretches from Hyderabad to the Arabian Sea, where the climate generally stays hot and dry in the daytime and the nights are calm and pleasant [19].

The field survey was conducted during the period from September 2018 to January 2019. For defining the sample size when the population is huge or the number is unidentified, the formula used by [19] was employed. As a final point, it was decided to manage 700 questionnaires to conduct this study. Moreover, cross-sectional household-level data was gathered from 700 respondents using the multi-stage stratified cluster sampling method (Table 1). In the first stage, districts were selected where various types of cooking fuels were used. In the second stage, from the selected districts, a further two sub-districts were selected. In the third stage, 10 villages from each sub-district were selected and 7 households from each village were interviewed face to face individually at each site using a standardized questionnaire. Apart from English, the questionnaire was orally translated into Sindhi (Local language) for the convenience of the general public.

\section{Variables and Their Specification}

\section{Dependent Variable}

Numerous solid fuels are used in the kitchen for cooking purposes, which has different levels of pollution and which affect/pollute the indoor air of the household, whereas [20] revealed the upper level of $\mathrm{PM}_{10}$ in the kitchen where solid fuels were used for cooking. Exposure to the concentration of pollution sequentially affects the health outcome [21]. However, the cooking fuels used were categorized as cleaner fuels and solid fuels and were grouped as a binary variable, whereas cleaner fuels like natural gas and electricity were indicated by "0". Solid cooking fuels like kerosene, wood, coal, agriculture waste, and dried animal dung were indicated by "1" in the dependent binary variable. Recently in the list of polluting fuels kerosene was added [22]. Therefore, kerosene is considered a polluting fuel as current research has revealed kerosene's highly positive effects on household air pollution [22]. For robustness we also estimate separate probit models using each cooking fuel type, described as a binary variable, that is, $\mathrm{F}=1$ if any polluting fuel is generally used for cooking, otherwise " 0 ".

\section{Independent Variables}

The socio-economic characteristics of the household indicate that age and education robustly affect household decisions [23]. In household attribute categories age, gender, education level, and occupation are used as (categorical) predictors which may affect the level of awareness and risks associated with exposure to indoor air pollution. Likewise, [24] indicated that unusually high ambient air pollution might arise from behaviors associated with age and/or co-exposures related to occupation. In addition, in this category we use cooking activities like households (HH) using a wood stove, cooking in the room, and indoor smoking as predictors for indoor air pollution. However, these predictors are grouped as cooking activities denoted by "C". Thus, for each activity a separate binary variable is demarcated. However, the preventing measures are taken to diminish exposure to pollution, and as a result, affects the health outcome [21]. In the present study, we use a living room with windows and the building structure has open windows for air ventilation, and the household has a separate kitchen outdoor for cooking. For each averting activity, a dummy variable is defined.

Many epidemiological studies have developed the relationship between ambient air pollution and health effects [25]. However, [26] revealed the vulnerable population clusters based on innate factors, integrated environmental, social or behavioral factors and unusually high exposures. Likewise, [27] depicted that long-term exposure to air pollution on Chinese students had a robust effect on their behavior and psychology along with their physical health. However, the behavioral health effects were recorded on a Likert scale for various behaviors, i.e., difficulty in concentration, feeling dry, dizziness, nausea, noticeable odors, highstress levels, fatigue/drowsiness, too hot temperature, and too cold temperature, which were grouped and denoted as " $B$ ".

Similarly, effects related to physical health were: sneezing/ blocked nose/sinus congestion, sore throat, eye irritation/contact lens problems, hyperventilation, shortness of breath, irritating cough/chest tightness, back pain/muscle twitching/aching joints, headache, heartburn, skin irritation/discolored skin/dry, and flaking skin. However, these physical effects were recorded on a Likert scale and then transformed into binary variables that are collectively denoted by " $P$ ". However, the data was collected from five districts of Sindh Province, namely Karachi, Hyderabad, Mirpur Khas, Shaheed Benazirabad, and Sukkur. While Karachi was kept as a reference district, and dummies were created for the other districts surveyed. Therefore, the regional disparity is denoted with " $R$ ", in which the districts capture the regional inequality between the districts. 
Table 1. Summary statistics of variables used for the probit regression model.

\begin{tabular}{|c|c|c|c|c|c|c|}
\hline & Unit & Description of variables & Mean & SD & Min & Max \\
\hline Dependent & Binary & If $\mathrm{HH}$ is using Solid Fuels used for cooking $=1$, otherwise 0 & 0.709 & 0.455 & 0 & 1 \\
\hline \multicolumn{7}{|c|}{ Independent Variable } \\
\hline \multirow{6}{*}{$\begin{array}{c}\text { Household } \\
\text { Attributes }\end{array}$} & Continuous & Age & 35.75 & 9.41 & 14 & 65 \\
\hline & Categorical & Gender & 1.445 & 0.500 & 1 & 2 \\
\hline & Categorical & Education & 2.099 & 1.739 & 0 & 4 \\
\hline & Continuous & Family Size & 6.427 & 1.611 & 3 & 13 \\
\hline & Continuous & HH income & 6,174 & 13,093 & 0 & 100,000 \\
\hline & Categorical & Occupation & 3.319 & 1.929 & 1 & 6 \\
\hline \multirow{3}{*}{$\begin{array}{l}\text { Cooking } \\
\text { Activities }\end{array}$} & Binary & If $\mathrm{HH}$ uses wood stove as heating system $=1$ else 0 & 0.547 & 0.498 & 0 & 1 \\
\hline & Binary & If the $\mathrm{HH}$ cooks indoor $=1$, otherwise 0 & 0.526 & 0.500 & 0 & 1 \\
\hline & Binary & If there is smoker in the $\mathrm{HH}=1$ otherwise 0 & 0.343 & 0.475 & 0 & 1 \\
\hline \multirow{3}{*}{$\begin{array}{l}\text { Averting } \\
\text { Activities }\end{array}$} & Binary & If the living area has windows $=1$, otherwise 0 & 0.804 & 0.397 & 0 & 1 \\
\hline & Binary & If building has open windows for air ventilation $=1$ else 0 & 0.513 & 0.500 & 0 & 1 \\
\hline & Binary & If $\mathrm{HH}$ has separate outdoor kitchen $=1$ otherwise 0 & 0.249 & 0.432 & 0 & 1 \\
\hline \multirow{9}{*}{$\begin{array}{l}\text { Behavioral } \\
\text { Health Effects }\end{array}$} & Binary & Difficulty in concentration & 0.647 & 0.478 & 0 & 1 \\
\hline & Binary & Feel Dry & 0.776 & 0.417 & 0 & 1 \\
\hline & Binary & Dizziness & 0.586 & 0.493 & 0 & 1 \\
\hline & Binary & Nausea & 0.549 & 0.498 & 0 & 1 \\
\hline & Binary & Noticeable odours & 0.809 & 0.394 & 0 & 1 \\
\hline & Binary & High stress levels & 0.307 & 0.462 & 0 & 1 \\
\hline & Binary & Fatigue/drowsiness & 0.873 & 0.333 & 0 & 1 \\
\hline & Binary & Temperature too hot & 0.974 & 0.158 & 0 & 1 \\
\hline & Binary & Temperature too cold & 0.563 & 0.496 & 0 & 1 \\
\hline \multirow{9}{*}{$\begin{array}{c}\text { Physical Health } \\
\text { Effects }\end{array}$} & Binary & Sneezing/ Blocked nose / Sinus congestion & 0.974 & 0.158 & 0 & 1 \\
\hline & Binary & Sore Throat & 0.927 & 0.260 & 0 & 1 \\
\hline & Binary & Eye irritation/ Contact lens problems & 0.973 & 0.163 & 0 & 1 \\
\hline & Binary & Hyperventilation, shortness of breath & 0.909 & 0.288 & 0 & 1 \\
\hline & Binary & Irritating cough/ chest tightness & 0.647 & 0.478 & 0 & 1 \\
\hline & Binary & Back pain/ Muscle twitching/ Aching joints & 0.831 & 0.375 & 0 & 1 \\
\hline & Binary & Headache & 0.320 & 0.467 & 0 & 1 \\
\hline & Binary & Heartburn & 0.440 & 0.497 & 0 & 1 \\
\hline & Binary & Skin irritation/discoloured skin/dry, flaking skin & 0.751 & 0.432 & 0 & 1 \\
\hline \multirow{4}{*}{$\begin{array}{l}\text { Regional } \\
\text { Disparity }\end{array}$} & Binary & Dummy Hyderabad & 0.200 & 0.400 & 0 & 1 \\
\hline & Binary & Dummy Shaheed Benazirabad & 0.200 & 0.400 & 0 & 1 \\
\hline & Binary & Dummy Mirpur khas & 0.200 & 0.400 & 0 & 1 \\
\hline & Binary & Dummy Sukkur & 0.200 & 0.400 & 0 & 1 \\
\hline
\end{tabular}


Table 2. Parameter estimates of Probit model and marginal effects for solid fuel heating.

\begin{tabular}{|c|c|c|c|c|}
\hline \multirow{2}{*}{ Variable } & \multicolumn{2}{|c|}{ Probit Estimates } & \multicolumn{2}{|c|}{ Marginal Effect } \\
\hline & Coef. & Std. Err. & Coef. & Std. Err. \\
\hline Cons & $2.408^{*}$ & 1.246 & & \\
\hline Age & -0.069 & 0.373 & -0.016 & 0.087 \\
\hline Gender & -0.026 & 0.172 & -0.006 & 0.040 \\
\hline Education & -0.039 & 0.080 & -0.009 & 0.019 \\
\hline Family size & 0.025 & 0.242 & 0.006 & 0.056 \\
\hline HH monthly income & 0.002 & 0.015 & 0.001 & 0.003 \\
\hline Occupation & 0.017 & 0.085 & 0.004 & 0.020 \\
\hline Wood stove for indoor heating & 0.414 & 0.167 & 0.097 & 0.038 \\
\hline Room cooking & -0.502 & 0.130 & -0.117 & 0.029 \\
\hline Smoker in $\mathrm{HH}$ & 0.036 & 0.164 & 0.008 & 0.038 \\
\hline Windows in Living area & $-0.647 * *$ & 0.210 & $-0.151 * *$ & 0.048 \\
\hline Building Air Condition & $0.116^{* * *}$ & 0.125 & $0.027 * * *$ & 0.029 \\
\hline HH has Separate Outdoor kitchen & 1.133 & 0.280 & 0.265 & 0.063 \\
\hline Difficulty in concentration & $-0.372 * * *$ & 0.173 & $-0.087 * * *$ & 0.040 \\
\hline Feel Dry & $0.381 * *$ & 0.184 & $0.089 * *$ & 0.042 \\
\hline Dizziness & $0.345^{* *}$ & 0.180 & $0.081 * *$ & 0.042 \\
\hline Nausea & $0.017 *$ & 0.207 & $0.004 *$ & 0.048 \\
\hline Noticeable odours & -0.279 & 0.175 & -0.065 & 0.041 \\
\hline High stress levels & $0.358^{*}$ & 0.195 & $0.084 *$ & 0.045 \\
\hline Fatigue/drowsiness & $0.631 * * *$ & 0.200 & $0.148 * * *$ & 0.046 \\
\hline Temperature too hot & -0.483 & 0.489 & -0.113 & 0.114 \\
\hline Temperature too cold & $-0.282 *$ & 0.145 & $-0.066^{*}$ & 0.034 \\
\hline Sneezing/ Blocked nose / Sinus congestion & $-0.958 * *$ & 0.437 & $-0.224 * *$ & 0.101 \\
\hline Sore Throat & -0.235 & 0.270 & -0.055 & 0.063 \\
\hline Eye irritation/ Contact lens problems & -0.259 & 0.464 & -0.060 & 0.108 \\
\hline Hyperventilation, shortness of breath & 0.005 & 0.234 & 0.001 & 0.055 \\
\hline Irritating cough/ Chest tightness & -0.008 & 0.168 & -0.002 & 0.039 \\
\hline Back pain/ Muscle twitching/ Aching joints & -0.014 & 0.199 & -0.003 & 0.047 \\
\hline Headache & -0.184 & 0.205 & -0.043 & 0.048 \\
\hline Heartburn & 0.268 & 0.207 & 0.063 & 0.048 \\
\hline Skin irritation/discoloured skin/dry, flaking skin & 0.231 & 0.166 & 0.054 & 0.039 \\
\hline Hyderabad & $-0.437 * *$ & 0.206 & $-0.102 * *$ & 0.048 \\
\hline Benazirabad & -0.305 & 0.210 & -0.071 & 0.049 \\
\hline Mirpur Khas & 0.390 & 0.275 & 0.091 & 0.064 \\
\hline Sukkur & $0.663 * *$ & 0.273 & $0.155^{* *}$ & 0.063 \\
\hline$N$ & 700 & & & \\
\hline Log-likelihood & -290.66 & & & \\
\hline Chi-square (34) & 263.47 & & & \\
\hline$F$-stat significance & 0.0000 & & & \\
\hline Pseudo- $R^{2}$ & 0.3 & & & \\
\hline
\end{tabular}




\section{Estimation Method and Econometric Model}

The model of the present study was developed on the basis of household health model, elaborated by [28], which was pioneered by [29] and was further improved by [30], keeping in mind the incidence that household health position possibly will be affected by indoor air pollution from the cooking fuels and other related factors. The household is health-related to the extent of pollution, which is defined in the dose-response function in the household health function in Equation (1). The dose-response function in its econometric model is written as;

$$
F=F(C, H, A, B, P, R+\varepsilon)
$$

...where $F$ is the output variable, which indicates the household health status who are using solid cooking fuels. Whereas household attributes $(H)$, cooking activities $(C)$, behavioral health effects $(B)$, physical health effects $(P)$, and the regional factors $(R)$. The error component $\varepsilon$ includes the component $\mu$ and the random error term $e . \mathrm{F}$ (.) is a function characterizing the values strictly from 0 and 1 . Furthermore, the variables are described and discussed as to how the dependent variable can be exaggerated by the exploratory variables derived in Equation (2).

$$
F_{i}^{*}=\left\{\begin{array}{l}
1 \text { if } F^{*}>0 \\
0 \text { otherwise }
\end{array}\right.
$$

The model in Equation (1) is estimated using the binary probit regression model. This method eliminates any underlying bias caused by variables omitted for the unobserved explanatory variables that may be associated with the other explanatory variables used in the model. Since the properties of large sample size, the maximum likelihood estimation method was employed to yield consistent coefficient estimates respectively.

\section{The Testing Model}

(Significance of the Hypothesis)

For examining the model significance a global null hypothesis was used in equation (3). For this study, a null hypothesis was developed assuming that all the coefficients are equal to zero of the binary Probit model versus that one coefficient is not zero [31].

$$
\begin{aligned}
& H_{0}: \\
& H_{I}: \text { at least } 1
\end{aligned}
$$

This method is like the $\mathrm{F}$ test in the ordinary least squares (OLS) technique, since the $\chi^{2}$ values for all seven models were positive and varied from 43 to 297 (Table 3). The related p-values are $<0.08$ for all the models. Hence, it can be determined that all the models fit significantly. Furthermore, the pseudo $R^{2}$ values (depicts the goodness of fit of a model) ranged from 0.16 to 0.38 , which indicates the good fit of our models in elucidating the physical and behavioral effect of cleaner and solid fuels in households toward the risk of polluting indoor air.

\section{Results and Discussion}

\section{Descriptive Statistics}

Table 1 reveals the descriptive statistics of the variables used for regression, which indicate that around $70 \%$ of households were using solid fuels for cooking purposes. Likewise, household attributes indicated that the majority of respondents formed the age group of 35 years, while the majority $(55.6 \%)$ of the respondents' gender was male. The findings coincide with [19]. Mostly education of the respondents was under graduation. However, the mean value of occupation reveals that the majority's occupation was housewife. Likewise, about $80 \%$ of households had windows in their living area. Moreover, 54\% were using a wood stove for heating purposes. Similar findings were also indicated by [32]. Similarly, around $51 \%$ had operable windows for air conditioning. However, more than $52 \%$ of households were cooking indoor and almost 25 percent had an outdoor kitchen. Around 34\% of households reported that there were smokers in the household, which are in line with [13]. However, the mean values of variables related to behavioral and physical health effects are depicted and also dummy's for a regional difference with reference to the Karachi district, in which, i.e., Hyderabad, Shaheed Benazirabad, Mirpur Khas and Sukkur districts are also portrayed in Table 1.

\section{Health Effects on Different Sites}

\section{Behavioral and Psychological Effects}

Fig. 2 reports the results of the study and shows respondents' perception regarding behavioral health effects of air pollution of study sites and an average of health effects of all districts. In the average of all districts, hot climatic conditions are marked as the major health effects of indoor air pollution in Sindh Province compared to cold weather. About $56 \%$ of respondents frequently felt too hot temperatures inside the building. Occasionally respondents feel difficulty in concentrating $(53.9 \%)$, tiredness/fatigue $(63.3 \%)$, high stress $(25.3 \%)$, dizziness $(48.7 \%)$, feeling dry $(61.4 \%)$ and nausea $(47.6 \%)$. Moreover, various research has indicated that long-term exposure to the toxic effects of indoor air pollution can increase psychological and behavioral problems, leading to anxiety, panic or fear, headache, nausea, fatigue, weakness, dizziness, insomnia, fainting, hyperventilation and nausea, and occasionally skin allergies, throat ache and eye irritation $[33,34]$. 
Table 3. Parameter estimates of Probit regression for cleaner and solid fuel for cooking and heating.

\begin{tabular}{|c|c|c|c|c|c|c|c|}
\hline Variables & Gas & Electricity & Kerosene & Wood & Coal & $\begin{array}{l}\text { Agri Crop } \\
\text { Waste }\end{array}$ & $\begin{array}{c}\text { Animal } \\
\text { Dung }\end{array}$ \\
\hline Age & $\begin{array}{c}0.044 \\
(0.097)\end{array}$ & $\begin{array}{l}-0.160 \\
(0.182)\end{array}$ & $\begin{array}{c}0.120 \\
(0.085)\end{array}$ & $\begin{array}{c}-0.052 \\
(0.098)\end{array}$ & $\begin{array}{c}-0.203 * \\
(0.122)\end{array}$ & $\begin{array}{c}0.013 \\
(0.136)\end{array}$ & $\begin{array}{c}0.035 \\
(0.137)\end{array}$ \\
\hline Education & $\begin{array}{c}0.028 \\
(0.082)\end{array}$ & $\begin{array}{c}0.038 \\
(0.137)\end{array}$ & $\begin{array}{c}0.059 \\
(0.073)\end{array}$ & $\begin{array}{c}-0.153 * * \\
(0.083)\end{array}$ & $\begin{array}{l}-0.086 \\
(0.110)\end{array}$ & $\begin{array}{l}-0.121 \\
(0.106)\end{array}$ & $\begin{array}{c}0.186 \\
(0.119)\end{array}$ \\
\hline Gender & $\begin{array}{c}0.081 \\
(0.179)\end{array}$ & $\begin{array}{c}-0.250 \\
(0.314)\end{array}$ & $\begin{array}{c}-0.317 * * \\
(0.169)\end{array}$ & $\begin{array}{c}0.248 \\
(0.217)\end{array}$ & $\begin{array}{c}0.064 \\
(0.235)\end{array}$ & $\begin{array}{l}-0.050 \\
(0.320)\end{array}$ & $\begin{array}{c}0.360 \\
(0.368)\end{array}$ \\
\hline Occupation & $\begin{array}{l}-0.026 \\
(0.085)\end{array}$ & $\begin{array}{c}0.065 \\
(0.151)\end{array}$ & $\begin{array}{c}0.050 \\
(0.077)\end{array}$ & $\begin{array}{l}-0.073 \\
(0.089)\end{array}$ & $\begin{array}{l}-0.123 \\
(0.115)\end{array}$ & $\begin{array}{l}-0.139 \\
(0.124)\end{array}$ & $\begin{array}{l}0.240 * \\
(0.139)\end{array}$ \\
\hline Family Size & $\begin{array}{c}0.171 \\
(0.253)\end{array}$ & $\begin{array}{l}-0.386 \\
(0.411)\end{array}$ & $\begin{array}{l}-0.144 \\
(0.222)\end{array}$ & $\begin{array}{c}0.276 \\
(0.255)\end{array}$ & $\begin{array}{c}0.168 \\
(0.318)\end{array}$ & $\begin{array}{c}-0.619^{*} \\
(0.349)\end{array}$ & $\begin{array}{c}0.027 \\
(0.350)\end{array}$ \\
\hline HH Income/ month & $\begin{array}{l}-0.011 \\
(0.014)\end{array}$ & $\begin{array}{c}0.029 \\
(0.026)\end{array}$ & $\begin{array}{l}-0.021 \\
(0.013)\end{array}$ & $\begin{array}{c}0.046^{* * *} \\
(0.017)\end{array}$ & $\begin{array}{c}0.021 \\
(0.019)\end{array}$ & $\begin{array}{c}-0.038^{*} \\
(0.023)\end{array}$ & $\begin{array}{c}0.021 \\
(0.027)\end{array}$ \\
\hline Air-condition & $\begin{array}{l}-0.142 \\
(0.129)\end{array}$ & $\begin{array}{c}0.230 \\
(0.234)\end{array}$ & $\begin{array}{c}0.085 \\
(0.115)\end{array}$ & $\begin{array}{c}0.144 \\
(0.140)\end{array}$ & $\begin{array}{c}-0.266 \\
(0.170)\end{array}$ & $\begin{array}{c}0.056 \\
(0.184)\end{array}$ & $\begin{array}{c}0.141 \\
(0.198)\end{array}$ \\
\hline Building Type & $\begin{array}{c}0.542 * * * \\
(0.213)\end{array}$ & $\begin{array}{c}0.593 \\
(0.449)\end{array}$ & $\begin{array}{c}0.355^{* *} \\
(0.171)\end{array}$ & $\begin{array}{c}-0.452 * * * \\
(0.153)\end{array}$ & $\begin{array}{c}0.012 \\
(0.228)\end{array}$ & $\begin{array}{c}0.001 \\
(0.213)\end{array}$ & $\begin{array}{c}-0.384 * * \\
(0.195)\end{array}$ \\
\hline Room cooking & $\begin{array}{c}0.598 * * * \\
(0.134)\end{array}$ & $\begin{array}{c}-0.595 * * \\
(0.256) \\
\end{array}$ & $\begin{array}{c}-0.577 * * * \\
(0.119)\end{array}$ & $\begin{array}{c}0.004 \\
(0.143) \\
\end{array}$ & $\begin{array}{l}-0.230 \\
(0.175) \\
\end{array}$ & $\begin{array}{c}0.112 \\
(0.187) \\
\end{array}$ & $\begin{array}{c}0.441 * * \\
(0.200)\end{array}$ \\
\hline Smoker & $\begin{array}{c}-0.025 \\
(0.174)\end{array}$ & $\begin{array}{l}-0.107 \\
(0.283)\end{array}$ & $\begin{array}{c}-0.273^{*} \\
(0.161)\end{array}$ & $\begin{array}{l}-0.099 \\
(0.196)\end{array}$ & $\begin{array}{c}-0.131 \\
(0.232)\end{array}$ & $\begin{array}{c}0.616^{* *} \\
(0.258)\end{array}$ & $\begin{array}{c}0.447 \\
(0.323)\end{array}$ \\
\hline Difficulty in concentration & $\begin{array}{c}0.335 * * \\
(0.160)\end{array}$ & $\begin{array}{c}-0.132 \\
(0.241)\end{array}$ & $\begin{array}{l}-0.219^{*} \\
(0.133)\end{array}$ & $\begin{array}{l}-0.082 \\
(0.153)\end{array}$ & $\begin{array}{c}0.014 \\
(0.182)\end{array}$ & $\begin{array}{l}0.390 * \\
(0.214)\end{array}$ & $\begin{array}{c}-0.073 \\
(0.216)\end{array}$ \\
\hline Feel Dryness & $\begin{array}{c}-0.356^{* *} \\
(0.157)\end{array}$ & $\begin{array}{c}0.184 \\
(0.254)\end{array}$ & $\begin{array}{c}0.042 \\
(0.132)\end{array}$ & $\begin{array}{l}-0.065 \\
(0.151)\end{array}$ & $\begin{array}{c}0.421 * * \\
(0.190)\end{array}$ & $\begin{array}{c}0.332 \\
(0.225)\end{array}$ & $\begin{array}{l}-0.047 \\
(0.224)\end{array}$ \\
\hline Dizziness & $\begin{array}{c}-0.461 * * * \\
(0.160)\end{array}$ & $\begin{array}{l}-0.064 \\
(0.249) \\
\end{array}$ & $\begin{array}{c}0.278 * * \\
(0.134)\end{array}$ & $\begin{array}{c}-0.139 \\
(0.159) \\
\end{array}$ & $\begin{array}{l}-0.066 \\
(0.185) \\
\end{array}$ & $\begin{array}{l}-0.004 \\
(0.214) \\
\end{array}$ & $\begin{array}{c}0.623 * * * \\
(0.244)\end{array}$ \\
\hline Nausea & $\begin{array}{l}-0.269 \\
(0.191)\end{array}$ & $\begin{array}{c}0.537 * * \\
(0.301)\end{array}$ & $\begin{array}{l}-0.180 \\
(0.170)\end{array}$ & $\begin{array}{l}-0.017 \\
(0.190)\end{array}$ & $\begin{array}{c}0.276 \\
(0.220)\end{array}$ & $\begin{array}{c}0.086 \\
(0.289)\end{array}$ & $\begin{array}{l}-0.272 \\
(0.28)\end{array}$ \\
\hline Noticeable odours & $\begin{array}{c}0.198 \\
(0.152) \\
\end{array}$ & $\begin{array}{c}-0.348 \\
(0.259) \\
\end{array}$ & $\begin{array}{c}-0.035 \\
(0.136) \\
\end{array}$ & $\begin{array}{c}-0.307 * * \\
(0.160)\end{array}$ & $\begin{array}{c}0.268 \\
(0.192) \\
\end{array}$ & $\begin{array}{c}-0.159 \\
(0.204) \\
\end{array}$ & $\begin{array}{c}0.065 \\
(0.242) \\
\end{array}$ \\
\hline High stress levels & $\begin{array}{l}-0.248 \\
(0.186)\end{array}$ & $\begin{array}{l}-0.053 \\
(0.279)\end{array}$ & $\begin{array}{c}0.060 \\
(0.149)\end{array}$ & $\begin{array}{c}0.035 \\
(0.169)\end{array}$ & $\begin{array}{c}0.136 \\
(0.192)\end{array}$ & $\begin{array}{l}-0.219 \\
(0.231)\end{array}$ & $\begin{array}{c}0.317 \\
(0.231)\end{array}$ \\
\hline Fatigue/drowsiness & $\begin{array}{c}-0.704 * * * \\
(0.142)\end{array}$ & $\begin{array}{c}-0.074 \\
(0.237) \\
\end{array}$ & $\begin{array}{c}0.524 * * * \\
(0.127)\end{array}$ & $\begin{array}{c}0.371 * * * \\
(0.151)\end{array}$ & $\begin{array}{c}0.206 \\
(0.181) \\
\end{array}$ & $\begin{array}{c}0.020 \\
(0.189) \\
\end{array}$ & $\begin{array}{c}-0.474 * * \\
(0.205)\end{array}$ \\
\hline Temperature too hot & $\begin{array}{c}0.385^{* *} \\
(0.160)\end{array}$ & $\begin{array}{l}-0.203 \\
(0.237)\end{array}$ & $\begin{array}{l}-0.126 \\
(0.136)\end{array}$ & $\begin{array}{l}-0.009 \\
(0.162)\end{array}$ & $\begin{array}{c}-0.068 \\
(0.200)\end{array}$ & $\begin{array}{c}0.190 \\
(0.221)\end{array}$ & $\begin{array}{c}0.207 \\
(0.250)\end{array}$ \\
\hline Temperature too cold & $\begin{array}{c}0.117 \\
(0.130) \\
\end{array}$ & $\begin{array}{c}0.238 \\
(0.209) \\
\end{array}$ & $\begin{array}{c}-0.257 * * \\
(0.114)\end{array}$ & $\begin{array}{c}-0.162 \\
(0.129) \\
\end{array}$ & $\begin{array}{l}-0.165 \\
(0.163) \\
\end{array}$ & $\begin{array}{c}0.129 \\
(0.170) \\
\end{array}$ & $\begin{array}{c}0.386 * * \\
(0.184)\end{array}$ \\
\hline $\begin{array}{l}\text { Sneezing/ Blocked nose / Sinus conges- } \\
\text { tion }\end{array}$ & $\begin{array}{c}0.166 \\
(0.141) \\
\end{array}$ & $\begin{array}{c}0.081 \\
(0.237) \\
\end{array}$ & $\begin{array}{c}-0.012 \\
(0.130) \\
\end{array}$ & $\begin{array}{r}-0.009 \\
(0.155) \\
\end{array}$ & $\begin{array}{l}-0.180 \\
(0.185)\end{array}$ & $\begin{array}{c}0.167 \\
(0.203) \\
\end{array}$ & $\begin{array}{l}-0.404 \\
(0.255)\end{array}$ \\
\hline Sore Throat & $\begin{array}{c}0.071 \\
(0.141) \\
\end{array}$ & $\begin{array}{l}-0.055 \\
(0.237)\end{array}$ & $\begin{array}{l}-0.041 \\
(0.126)\end{array}$ & $\begin{array}{c}0.181 \\
(0.146) \\
\end{array}$ & $\begin{array}{c}0.056 \\
(0.182)\end{array}$ & $\begin{array}{l}-0.393 * \\
(0.205)\end{array}$ & $\begin{array}{l}-0.094 \\
(0.210)\end{array}$ \\
\hline Eye irritation/ Contact lens problems & $\begin{array}{c}0.145 \\
(0.138) \\
\end{array}$ & $\begin{array}{l}-0.204 \\
(0.241) \\
\end{array}$ & $\begin{array}{l}-0.076 \\
(0.126) \\
\end{array}$ & $\begin{array}{c}-0.131 \\
(0.151) \\
\end{array}$ & $\begin{array}{l}0.356^{*} \\
(0.203)\end{array}$ & $\begin{array}{l}-0.393 * \\
(0.219)\end{array}$ & $\begin{array}{c}0.186 \\
(0.239)\end{array}$ \\
\hline Hyperventilation, shortness of breath & $\begin{array}{c}0.019 \\
(0.140) \\
\end{array}$ & $\begin{array}{c}0.105 \\
(0.248) \\
\end{array}$ & $\begin{array}{c}0.074 \\
(0.128) \\
\end{array}$ & $\begin{array}{r}-0.098 \\
(0.159) \\
\end{array}$ & $\begin{array}{l}-0.127 * \\
(0.185)\end{array}$ & $\begin{array}{c}0.047 \\
(0.238) \\
\end{array}$ & $\begin{array}{l}-0.420 \\
(0.243)\end{array}$ \\
\hline Irritative cough/ Chest tightness & $\begin{array}{c}0.019 \\
(0.138) \\
\end{array}$ & $\begin{array}{c}-0.024 \\
(0.216) \\
\end{array}$ & $\begin{array}{c}-0.124 \\
(0.121)\end{array}$ & $\begin{array}{c}0.090 \\
(0.145) \\
\end{array}$ & $\begin{array}{c}0.272 \\
(0.166)\end{array}$ & $\begin{array}{c}-0.148 \\
(0.195) \\
\end{array}$ & $\begin{array}{l}-0.168 * \\
(0.206)\end{array}$ \\
\hline $\begin{array}{l}\text { Back pain / Muscle twitching / Aching } \\
\text { joints }\end{array}$ & $\begin{array}{c}0.140 \\
(0.131)\end{array}$ & $\begin{array}{c}0.266 \\
(0.229)\end{array}$ & $\begin{array}{l}-0.015 * * \\
(0.119)\end{array}$ & $\begin{array}{l}-0.358 \\
(0.147)\end{array}$ & $\begin{array}{c}0.206^{* *} \\
(0.176)\end{array}$ & $\begin{array}{l}-0.202 \\
(0.186)\end{array}$ & $\begin{array}{c}0.387 \\
(0.198)\end{array}$ \\
\hline Headache & $\begin{array}{l}0.124 \\
(0.175)\end{array}$ & $\begin{array}{l}-0.163 \\
(0.285)\end{array}$ & $\begin{array}{l}-0.358 \\
(0.157)\end{array}$ & $\begin{array}{l}0.339 * * \\
(0.167)\end{array}$ & $\begin{array}{l}-0.427 \\
(0.209)\end{array}$ & $\begin{array}{c}0.314 \\
(0.222)\end{array}$ & $\begin{array}{c}-0.039 * * \\
(0.242)\end{array}$ \\
\hline
\end{tabular}


Table 3. Continued.

\begin{tabular}{|c|c|c|c|c|c|c|c|}
\hline Heartburn & $\begin{array}{l}-0.068 \\
(0.190)\end{array}$ & $\begin{array}{l}-0.250 \\
(0.269)\end{array}$ & $\begin{array}{c}0.097 \\
(0.157)\end{array}$ & $\begin{array}{c}0.270 * * \\
(0.188)\end{array}$ & $\begin{array}{l}-0.205 \\
(0.204)\end{array}$ & $\begin{array}{c}0.044 \\
(0.276)\end{array}$ & $\begin{array}{c}0.204 \\
(0.288)\end{array}$ \\
\hline $\begin{array}{l}\text { Skin irritation/ Discolored skin/ Dry, } \\
\text { flaking skin }\end{array}$ & $\begin{array}{c}-0.262 * * * \\
(0.119)\end{array}$ & $\begin{array}{l}-0.005 \\
(0.199)\end{array}$ & $\begin{array}{c}0.172 \\
(0.108)\end{array}$ & $\begin{array}{l}-0.047 \\
(0.129)\end{array}$ & $\begin{array}{l}-0.061 \\
(0.159)\end{array}$ & $\begin{array}{l}-0.157 \\
(0.176)\end{array}$ & $\begin{array}{l}0.330 * \\
(0.189)\end{array}$ \\
\hline Hyderabad & $\begin{array}{l}-0.091 \\
(0.188)\end{array}$ & $\begin{array}{l}0.669 * \\
(0.409)\end{array}$ & $\begin{array}{l}-0.015 \\
(0.175)\end{array}$ & $\begin{array}{c}0.040 \\
(0.354)\end{array}$ & $\begin{array}{c}-0.899 * * * \\
(0.311)\end{array}$ & $\begin{array}{c}4.034 \\
(187.446)\end{array}$ & -- \\
\hline Benazir Abad & $\begin{array}{c}-0.242 \\
(0.193)\end{array}$ & $\begin{array}{c}0.566 \\
(0.410)\end{array}$ & $\begin{array}{l}-0.208 \\
(0.177)\end{array}$ & $\begin{array}{c}0.547 * * \\
(0.301)\end{array}$ & $\begin{array}{l}-0.230 \\
(0.253)\end{array}$ & $\begin{array}{c}4.227 \\
(187.446)\end{array}$ & $\begin{array}{c}4.666 \\
(190.206)\end{array}$ \\
\hline Mirpur Khas & $\begin{array}{l}-1.426 * * * \\
(0.240)\end{array}$ & $\begin{array}{c}0.188 \\
(0.486)\end{array}$ & $\begin{array}{l}-0.959 * * * \\
(0.203)\end{array}$ & $\begin{array}{c}1.553 * * * \\
(0.278)\end{array}$ & $\begin{array}{l}-0.459 * \\
(0.273)\end{array}$ & $\begin{array}{c}5.297 \\
(187.446)\end{array}$ & $\begin{array}{c}5.830 \\
(190.206)\end{array}$ \\
\hline Sukkur & $\begin{array}{l}-1.768 * * * \\
(0.276)\end{array}$ & $\begin{array}{c}0.275 \\
(0.481)\end{array}$ & $\begin{array}{l}-0.991 * * * \\
(0.206)\end{array}$ & $\begin{array}{c}1.682 * * * \\
(0.274)\end{array}$ & $\begin{array}{c}-0.764 * * \\
(0.306)\end{array}$ & $\begin{array}{c}4.752 \\
(187.446)\end{array}$ & $\begin{array}{c}6.150 \\
(190.205)\end{array}$ \\
\hline Intercept & $\begin{array}{l}-0.729 \\
(0.766)\end{array}$ & $\begin{array}{l}-1.369 \\
(1.365)\end{array}$ & $\begin{array}{c}0.112 \\
(0.683)\end{array}$ & $\begin{array}{l}-1.518 \\
(0.802)\end{array}$ & $\begin{array}{c}-2.365 * * \\
(1.053)\end{array}$ & $\begin{array}{c}-4.293 \\
(187.449)\end{array}$ & $\begin{array}{c}-9.848 \\
(190.210)\end{array}$ \\
\hline$N$ & 700 & 700 & 700 & 700 & 700 & 700 & 560 \\
\hline Log likelihood & -268.19 & -79.265 & -342.93 & -240.79 & -149.87 & -136.95 & -131.25 \\
\hline$C h i^{2}(33)$ & 0.000 & 0.0795 & 0.000 & 0.000 & 0.0001 & 0.000 & 0.000 \\
\hline$F$-stat significance & 267.99 & 43.82 & 138.55 & 178.37 & 70.67 & 96.53 & 163.35 \\
\hline Pseudo- $R^{2}$ & 0.3332 & 0.2166 & 0.1681 & 0.2703 & 0.1908 & 0.2606 & 0.3836 \\
\hline
\end{tabular}

$* * *, * *, *$ significant at significance level $0.01,0.05$ and 0.1 respectively

\section{Physical Health Effects}

Fig. 3 divulges the results of the study and shows respondents' perception regarding the physical health effects of air pollution of study sites and an average of health effects of all districts. Respondents were well aware of possible symptoms and health effects of exposure to indoor air pollution. The complaints mainly referred to irritation of eyes and nose, marked as major health effects in all districts of Sindh Province, Pakistan. Irritation of eyes and nose occur frequently in $51.4 \%$ and $66.1 \%$ of respondents respectively. Similarly, [33] indicated that ophthalmic symptoms were more prevalent (i.e., itchy, dry and irritated eyes and lightheadedness or dizziness, etc.). Headache, throat ache, cough, breathing problems and skin allergies being noted occasionally. However, headache, throat, cough, breathing problems and skin allergy occured in $54.3 \%, 54.1 \%, 53.9 \%, 50.0 \%$, and $49.7 \%$ of respondents respectively. Other problems affect the health of a human being to a lesser extent, such as back (25.7\% of respondents) and heartburn (in $37.4 \%$ of respondents occasionally). In a previous study, [17] reported physical health problems including coughing, wheezing, chest pain, headache, shortness of breath, and irritation in eyes due to poor indoor air quality in most rural households.

Table 2 depicts the results revealed from probit regression indicating that solid cooking fuels have a statistically significant effect on polluting indoor household air. However, results indicate that the coefficient of age was negative but highly significant

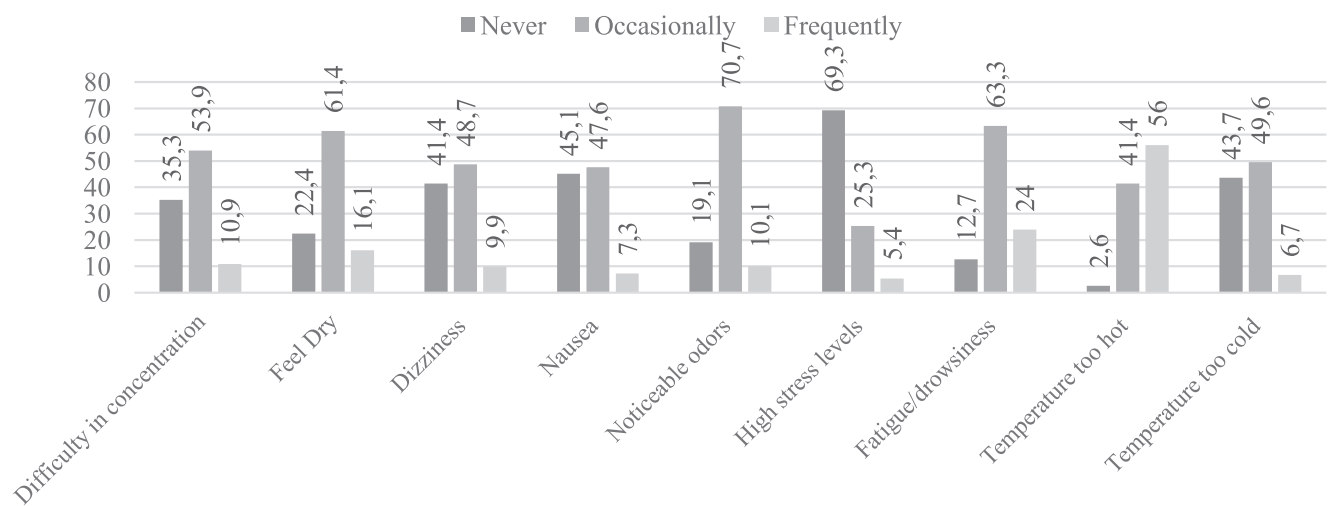

Fig. 2. Behavioral and psychological effects. 


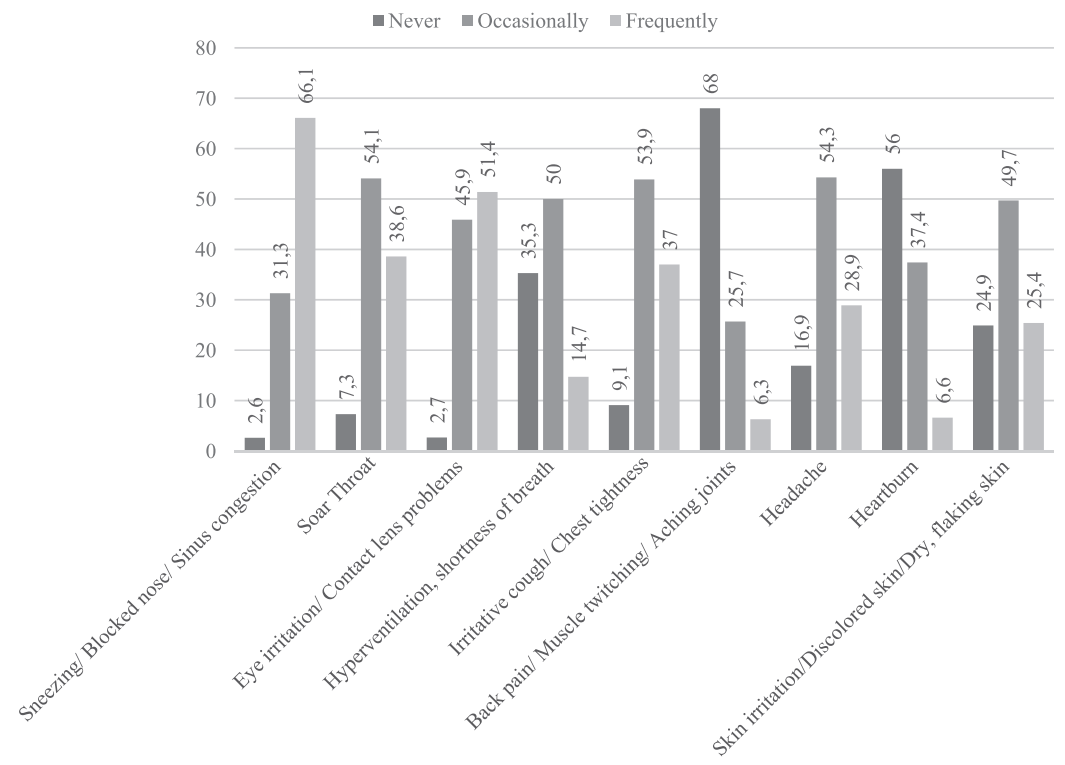

Fig. 3. Physical health effects.

with solid fuels used for cooking. Hence, it could be elaborated that age may decrease due to the polluting effect of solid fuel usage. These findings are in line with [28]. Likewise, the coefficient of gender (0.126) also revealed a significantly positive correlation with households using solid fuel. Moreover, the coefficient of household income was a significant and positive correlation with solid fuel. This may be due to different levels of income. Moreover, the coefficient of the living area was negative but significant, which indicates that living areas with windows helps to combat indoor air pollutants. Similarly, the coefficient of room cooking was negative and significant. Similarly, the coefficient of the outdoor kitchen was negative and significant.

Likewise, [35] indicated that females were more sensitive than males when exposed to air pollutants. When exposed to $\mathrm{PM}_{10}$, males were at higher risk of having cardiovascular admission than females in Vietnam. However, the coefficient of feeling dry was negative and significant. Hence the marginal effects indicate that a $1 \%$ change in feeling dry behavior would decrease by $5.3 \%$. However, the coefficient of nausea is a positive and significant effect on behavioral health. However, the marginal effect indicates that a $1 \%$ change in solid fuel usage would change the effect of nausea by $12.5 \%$. Similarly, [36] indicated that nausea occurred in $0.41 \%$ of respondents in India. Moreover, the coefficient of the high-stress level was positive and significant. This indicates that a $1 \%$ change in high-stress level would change the stress level by $11.1 \%$. Similarly, findings are in line with [36], who also reported a high-stress level of $1.97 \%$ in Gwalior India. Moreover, the coefficient fatigue/drowsiness was negative but highly significant. Likewise, the coefficient of too cold temperature was positive and highly significant. Thus, the marginal effects indicate that a $1 \%$ change would increase the risk of being affected by too cold temperature by $8.2 \%$. The coefficient of sinus congestion was highly significant and negative. This indicates that a $1 \%$ change in usage of solid fuel may change the risk of sinus congestion by $9.2 \%$. Moreover, the coefficient of irritating cough was positive and significant, indicating that a $1 \%$ change in usage of solid fuel may change the risk of irritating cough by $3.2 \%$. Thus these findings are in line with [28]. However, the coefficients of regional disparity for Mirpur Khas and Sukkur districts were negative but significant respectively. Probit model parameter estimates for clean and solid fuels

For viewing the effects of different fuels used for cooking, an individual probit model was driven and revealed in Table 3 . The selected variables driven in the model were on the basis that at least it was significant in one driven model. Results reveal that the coefficient of the age of respondent is significant and negative for households using coal for cooking purposes. This indicates that a $1 \%$ increase in age would decrease the probability of polluting effect from cooking. Similarly, the coefficient of education was significant and negative for the households using wood as a solid fuel for cooking. This divulges that a $1 \%$ increase in education would decrease the probability of polluting from using wood for cooking. The coefficient of gender was significant and negative for the households using kerosene, which indicates that a $1 \%$ change in gender would decrease the probability of polluting from using kerosene for cooking. Similarly, [28] indicated that kerosene was used occasionally for cooking, such as making tea, etc. However, the coefficient occupation was significant and positive, indicating that a $1 \%$ increase in the occupation will increase the probability of polluting by using animal dung for cooking. However, the coefficient of family size was significant and negative 
for the user's agricultural crop waste. This indicates that a $1 \%$ increase in family size would decrease the probability of polluting by using agriculture crop waste for cooking. However, the coefficient of $\mathrm{HH}$ income was significant and positive for wood and negative for agriculture crop wastage users. This divulges that a $1 \%$ change in $\mathrm{HH}$ income would decrease the probability of using agricultural crop waste and increase the possibility of using wood for the purpose of cooking. However, [37] divulging that low-income households are highly exposed to indoor air pollution because of using unclean fuel for cooking. Likewise, the coefficient of building type was positively and negatively significant for gas, kerosene, wood and animal dung, respectively.

Similarly, [32] revealed that in Pune India, 45\% of households had no separate kitchen for cooking. However, the coefficient of indoor cooking was revealed to be significant for gas, electricity, kerosene and animal dung. Similarly, the coefficient of the smoker was found to be significant in households using kerosene and agriculture crop wastage for cooking. Similarly, [13] reported that the concentration of $\mathrm{PM}_{2.5}$ and $\mathrm{PM}_{1}$ indicated less fluctuation where the living rooms were occupied with smokers. Likewise, the coefficient of difficulty in concentration was significant for gas, kerosene, and agricultural crop waste users. This indicates that a $1 \%$ change in fuel usage would increase or decrease polluting indoor household air. Moreover, the coefficient of feeling dry revealed significant and positive association for gas and coal, which indicates that a $1 \%$ increase in coal usage would increase the probability of polluting by using coal. However, findings are in line with [33]. Similarly, a 1\% increase in gas usage would decrease the probability of feeling dry. However, the coefficient of dizziness was negative and significant for gas and positive for kerosene and animal dung. However, findings coincide with [33]. This indicates that a $1 \%$ increase in the use of gas will decrease the probability of dizziness by $46 \%$. Moreover, a $1 \%$ increase in the use of kerosene and animal dung usage would increase the probability of having dizziness effect by $27 \%$ and $62 \%$. Similarly, [38] revealed that the patient also reported feeling dizziness while cooking.

Furthermore, the coefficient of nausea was significant and positive, indicating that a $1 \%$ increase in the use of electricity would increase nausea by $53 \%$. The coefficient of the noticeable odor was negative and significant for households using wood fuel. The coefficient of fatigue/drowsiness was significant for gas, kerosene, wood and animal dung. However, a 1\% increase in the use of gas may decrease the probability of feeling drowsy. Similarly, a $1 \%$ increase in the use of kerosene and wood may increase the chance of having a fatigue/drowsiness effect. However, the coefficient of too hot temperature was significant for gas, indicating that a $1 \%$ increase in use of gas may increase the probability of temperatures being too hot by $38 \%$. Similarly, the coefficient of too cold temperature was significant and negative for households using kerosene and animal dung for cooking. This indicates that a $1 \%$ increase in the use of kerosene would decrease the probability of being too cold by $25 \%$, and a $1 \%$ increase in the use of animal dung would increase the probability of indoor air being polluted. Likewise, the coefficient of sore throat was significant and negative, indicating that increasing the use of agricultural crop waste would negatively affect a sore throat. Likewise, the coefficient of eye irritation was significant and negative, indicating that increasing the use of crop waste would negatively affect eye irritation by 39\%. Likewise, [17] and [38] divulged that women also complained of itching and tears in the eyes while cooking. Moreover, the coefficient of hyperventilation/shortness of breath was significant and negative, indicating that a $1 \%$ increase in the use of coal would negatively affect hyperventilation/ shortness of breath by $12 \%$. However, [39] divulged that subsistence farmers almost exclusively use traditional open-fire stoves for cooking, and the majority of rural kitchens are made of adobe without any chimney or ventilation.

Dense black smoke stains surrounding the opening of the stove and on the walls imply the level of pollutants the household members breathe in as a result of the stove. Furthermore, the coefficient of irritating cough was significant and negative for animal dung users. This divulges that a $1 \%$ increase in animal dung usage would negatively affect irritative coughs by around $16 \%$. However, [40] indicated that one-third of vendors were aware that respiratory problems such as cough and breathing problems are caused by biomass fuel smoke. Moreover, the coefficient of aching joints was significantly positive and negative. Therefore, this indicates that a $1 \%$ increase in usage of kerosene would negatively affect aching joints and a $1 \%$ increase in coal would positively affect aching joints. According to [17], due to the use of a traditional cooking stove, $70 \%$ of respondents reported a complaint of chest pain and difficulty in breathing. Also, [3] indicated that patients also reported having body aches. Furthermore, the coefficient of headache was significant and positive for wood fuel users and negative for animal dung users. Hence, this indicates that a $1 \%$ increase in woodfuel use would probably increase the chance of having a headache. Likewise, a $1 \%$ increase in the usage of animal dung may negatively affect persons with a headache. Similarly, [3] divulged that exposure to biomass fuel smoke is significantly connected with the prevalence of headache symptoms. However, the coefficient of skin irritation revealed a significant but negative association with households using gas for cooking purposes. However, the coefficients of districts indicate significantly positive and negative associations with clean and solid fuels used for households cooking indoors.

The current study has some limitations on its part such as time, funding, instrument availability, and management of traveling across sampling sites. The other limitations include missing a detailed investigation 
on the interaction of different air pollutants, which is rarely available in other such studies as well. For future research, studies in other provinces/parts of Pakistan, it is suggested that the exposure duration, air pollutant concentrations, total ventilation, hospital admissions if any, and outdoor concentrations of the pollutants should be recorded. Furthermore, the epidemiological approach should be adopted along with the ecological one to make the studies more intact and reliable. Intraindividual variations of the perceptions should be taken into consideration, too.

Air pollution has been a horrible issue for Pakistan recently. Therefore, mass awareness should be included as an integral part of campaigns against it. The local, provincial, and central governments should launch a program to educate people across the country regarding the adverse affects of pollution, precautionary measures to be adapted, and how to avoid it. The environmental protection agencies should install air pollution recording systems across the country and regularly update the subjects regarding an increase in concentrations of the pollutants. This will not only help to avoid them or be more cautious about them but also portray a message about how important and key an issue this is. Furthermore, the government should install purification systems like in China. An agricultural campaign should be an integral and key part of any environmental ministry plans. "Go Green Pakistan" or "Green Pakistan, Green Future" are possible slogans for the coming decade or two.

\section{Conclusions}

The aim of this study was to assess the impact of indoor air pollution on households that were directly or indirectly involved in the use of fuels (cleaner and solid) for cooking purposes. Our study spans a large area and the effects were observed between and within the districts to obtain a holistic picture of indoor air pollution impact on households. In the study area, 70\% of households accounted for the consumption of solid fuels for cooking purposes. Likewise, age, education, gender, occupation, family size, and income are significant factors, which were affecting the usage of solid fuel for cooking. However, averting activities play a significant role in reducing and controlling indoor air pollution. The majority of households had opening windows in their living areas and kitchens, and only one-fourth households had separate kitchens outdoors, and a significant relationship was determined between behavioral and physical health effects for the households using cleaner/solid fuels. It is likely that the burden of disease may increase from the household's indoor air pollution if measures are not taken to mitigate the scenario. Furthermore, there was a significant regional disparity between households using various fuels for cooking. To mitigate these effects, awareness programs to educate rural households about proper ventilation for their kitchens are needed. Also, alternative energy sources may be provided additionally for preventing the adverse effect of solid fuel combustion. Improving the indoor air pollution in rural households' proper gas stoves should be used in the kitchen and if impossible improved stoves should be developed and promoted for burning of biomass with the least emission of fuel gases.

\section{Acknowledgements}

Author S.A. Rajper was supported by the Chinese Scholarship Council and National Natural Science Foundation of China (No. 31772470) for his Ph.D. study. We are thankful to all the respondents who participated in this study.

\section{Conflict of Interest}

The authors declare no conflict of interest.

\section{References}

1. BONJOUR S., ADAIR-ROHANI H., WOLF J., BRUCE N.G., MEHTA S., PRÜSS-USTÜN A., SMITH K. R. Solid fuel use for household cooking: country and regional estimates for 1980-2010. Environmental health perspectives. 121 (7), 784, 2013.

2. BHANDARKAR S. Vehicular Pollution, Their Effect on Human Heatlh and Mitigation Measures. Vehicle Engineering (VE). 1 (2), 33, 2013.

3. MOHAPATRA I., DAS S.C., SAMANTARAY S. Health impact on women using solid cooking fuels in rural area of Cuttack district, Odisha. Journal of family medicine and primary care. 7 (1), 11, 2018.

4. SAHA A., ATUL A., HIRA S., NASKAR N.N.,LAHIRI T.K. Association of adverse neonatal outcome with biomass fuel use. Environmental Disease. 2 (2), 55, 2017.

5. SAHA A., ROY S., YADAV A., MUKHERJEE A. A study of environmental exposure from cooking fuel use and role of intervention. Environmental Disease. 3 (3), 69, 2018.

6. JELILI M.O., GBADEGESIN A.S., ALABI A.T. Intraurban Pattern and Land Use Determinants of Outdoor Air Quality in Ogbomoso, Nigeria. Journal of Civil and Environmental Studies. 2 (1), 30, 2019.

7. PBS. Pakistan Bureau of Statistics. Provisional Summary Results of $6^{\text {th }}$ Population and Housing Census - 2017, Islamabad. 2017.

8. GOP. Goverment of Pakistan. Economics Affairs Division. Economic Survey of Pakistan, Islamabad. 2018.

9. NAZ S., PAGE A., AGHO K.E. Household air pollution from use of cooking fuel and under-five mortality: The role of breastfeeding status and kitchen location in Pakistan. PLoS ONE. 12 (3), 1, 2017.

10. BHUTTO A.W., BAZMI A.A., ZAHEDI G. Greener energy: Issues and challenges for Pakistan - Biomass energy prospective. Renewable and Sustainable Energy Reviews. 15 (6), 3207, 2011. 
11. NIPS. Demographic and Health survey. National Institute of Population Studies Islamabad, Government of Pakistan, Islamabad. 2018.

12. JANJUA N.Z., MAHMOOD B., DHARMA V.K., SATHIAKUMAR N., KHAN M.I. Use of biomass fuel and acute respiratory infections in rural Pakistan. Public health. 126 (10), 855, 2012.

13. NASIR Z.A., COLBECK I., ALI Z., AHMAD,S. Indoor particulate matter in developing countries: A case study in Pakistan and potential intervention strategies. Environmental Research Letters. 8 (2), 2013.

14. GORDON P.S.B. Europe PMC Funders Group Respiratory risks from household air pollution in low and middle income countries. 2 (10), 823, 2016.

15. CHAKRABORTY D., MONDAL N.K., DATTA J.K. Indoor pollution from solid biomass fuel and rural health damage: A micro-environmental study in rural area of Burdwan, West Bengal. International Journal of Sustainable Built Environment. 3 (2), 262, 2014.

16. KUMAR R., SINGH K., NAGAR S., KUMAR M., MEHTO U.K., RAI G., GUPTA N. Pollutant levels at cooking place and their association with respiratory symptoms in women in a rural area of Delhi-NCR. Indian J Chest Dis Allied Sci. 57 (4), 225, 2015.

17. PARAJULI I., LEE H., SHRESTHA K.R. Indoor Air Quality and ventilation assessment of rural mountainous households of Nepal. International Journal of Sustainable Built Environment. 5 (2), 301, 2016.

18. WIKIPEDIA. List of Pakistani provinces by gross domestic product. Retrieved from https://en.wikipedia.org/ wiki/List of Pakistani provinces by gross domestic product\#Provinces by GDP .28nominal.29. 2019.

19. NAZIR A., LI G., INAYAT S., IQBAL M.A., HUMAYOON A., AKHTAR S. Determinants for Income Diversification by Farm Households in. The Journal of Animal and Plant Sciences. 28 (4), 1, 2018.

20. BRUCE N., POPE D., REHFUESS E., BALAKRISHNAN K., ADAIR-ROHANI H., DORA C. WHO indoor air quality guidelines on household fuel combustion: Strategy implications of new evidence on interventions and exposure-risk functions. Atmospheric Environment. 106, 451, 2015.

21. FREEMAN M.A., HERRIGES J.A., KLING C.L. The Measurement of Environmental Resource Values: Theory and Methods. The Measurement of Environmental Resource Values. Retrieved from http://econdse.org/wpcontent/uploads/2016/07/Freeman-Herriges-Kling-2014. pdf. 2014.

22. WHO. World Health Organization. Burning Opportunity: Clean Household Energy for Health, Sustainable Development, and Wellbeing of Women and Children. 2016.

23. ABID M., SCHILLING J., SCHEFFRAN J., ZULFIQAR F. Climate change vulnerability, adaptation and risk perceptions at farm level in Punjab, Pakistan. Science of the Total Environment. 547, 447, 2016.

24. MAKRI A., STILIANAKIS N.I. Vulnerability to air pollution health effects. International journal of hygiene and environmental health. 211 (3-4), 326, 2008

25. ÖZKAYNAK H., BAXTER L.K., DIONISIO K.L., BURKE J. Air pollution exposure prediction approaches used in air pollution epidemiology studies. Journal of Exposure Science and Environmental Epidemiology. 23 (6), 566, 2013
26. WHO. World Health Organization. Situation Analysis of Household Energy Use and Indoor Air Pollution in Pakistan. 2005.

27. RAJPER S.A., ULLAH S., LI Z. Exposure to air pollution and self-reported effects on Chinese students: A case study of 13 megacities. PLOS ONE. 13 (3), 2018.

28. KHAN M.S.B., LOHANO H.D. Household air pollution from cooking fuel and respiratory health risks for children in Pakistan. Environmental Science and Pollution Research. 25 (25), 24778, 2018.

29. GROSSMAN M. On the concept of health capital and the demand for health. Journal of Political economy. 80 (2), 223, 1972

30. HARRINGTON W., PORTNEY P.R. Valuing the benefits of health and safety regulation. Journal of urban Economics. 22 (1), 101, 1987.

31. PING Q., IQBAL M.A., ABID M., AHMED U.I., NAZIR A., REHMAN A. Adoption of Off-farm Diversification Income Sources in Managing Agricultural Risks among Cotton Farmers in Punjab Pakistan. J. Appl. Environ. Biol. Sci, 6 (8), 47, 2016

32. ELF J.L., KINIKAR A., KHADSE S., MAVE V., SURYAVANSHI N., GUPTE N., GOLUB J.E. Sources of household air pollution and their association with fine particulate matter in low-income urban homes in India. Journal of Exposure Science \& Environmental Epidemiology. 2018.

33. NORHIDAYAH A., LEE C.K., AZHAR M.K., NURULWAHIDA S. Indoor air quality and sick building syndrome in three selected buildings. Procedia Engineering. 53 (2010), 93, 2013.

34. ŽIVKOVIĆ S., VELJKOVIĆ M. Psychological effects of indoor air pollution. Facta Universitatis, Series: Working and Living Environmental Protection. 11 (2), 109, 2015.

35. PHUNG D., HIEN T.T., LINH H.N., LUONG L.M.T., MORAWSKA L., CHU C., THAI P.K. Air pollution and risk of respiratory and cardiovascular hospitalizations in the most populous city in Vietnam. Science of The Total Environment. 557, 322, 2016.

36. SHARMA H.K., JADON N., DANDOTIYA B. Exposure of Air Pollution and Its Health Effects in Traffic Police Persons of Gwalior City, India. Environmental Claims Journal. 2017.

37. NASIR Z.A., MURTAZA F., COLBECK I. Role of poverty in fuel choice and exposure to indoor air pollution in Pakistan. Journal of Integrative Environmental Sciences. 12 (2), 107, 2015.

38. DHARMARAJU N., MAULESHBHAI S.S., ARULAPPAN N., THOMAS B., MARCONI D.S., PAUL S.S., MOHAN V.R. Household food security in an urban slum: Determinants and trends. Journal of Family Medicine and Primary Care. 7 (1), 819, 2018.

39. POLLARD S.L., WILLIAMS D.L., BREYSSE P.N, BARON P.A., GRAJEDA L.M., GILMAN R.H., CHECKLEY W. A cross-sectional study of determinants of indoor environmental exposures in households with and without chronic exposure to biomass fuel smoke. Environmental Health: A Global Access Science Source. 13 (1), 1, 2014.

40. NWANKWO O.N.O., MOKOGWU N., AGBOGHOROMA O., AHMED F.O., MORTIMER, K. Knowledge, attitudes and beliefs about the health hazards of biomass smoke exposure amongst commercial food vendors in Nigeria. PLoS ONE. 13 (1), 1, 2018. 